\title{
A Numerical Study of Spontaneous Propane Ignition in a Partially Confined Volume
}

\author{
CAROLYN R. KAPLAN
}

Navy Technology Center for Safety and Survivability

Naval Research Laboratory, Washington, DC 20375, USA

ELAINE S. ORAN

Laboratory for Computational Physics and Fluid Dynamics

Naval Research Laboratory, Washington, DC 20375, USA

\begin{abstract}
Numerical simulations are presented of the spontaneous ignition of a stoichiometric propane-air mixture in a duct containing an obstacle. The simulations were performed by combining a solution of the compressible equations of fluid dynamics with a phenomenological chemical induction model for species conversion and energy release. The mixture ignites as a weak shock wave hits and is reflected from the obstacle. Nonreactive simulations show how the obstacle partially blocks the flow such that one portion of the shock front reflects off of the obstacle and another portion is transmitted. Reactive-flow simulations show spontaneous ignition in the reflected shock region, which quickly transitions to a detonation. Eventually, transition to detonation also occurs in the transmitted shock direction. As the obstacle height is increased, the strength of the transmitted wave is decreased and transition to detonation requires longer time.
\end{abstract}

KEYWORDS: numerical simulation, spontaneous ignition, fuel-air explosions

\section{INTRODUCTION}

Fires resulting from the accidental leakage of hydrocarbon fuels can have devastating consequences. Usually these leaks occur in confined environments where there are obstacles with complex shapes. The presence of obstacles leads to an increase in the rate of burning and acceleration of the flame front, and, in many circumstances, may be responsible for producing violent explosions. Indeed, a wide variety of both large- and small-scale experiments [1-5] have demonstrated the dramatic influence of confinement and the presence of obstacles on the severity of fuel-air explosions.

This paper reports a numerical study of spontaneous ignition of a stoichiometric propane-air mixture in a partially confined volume. The physical 
scenario represents a situation in which the propane has leaked from a storage container and has been uniformly distributed throughout the simulated area. Then a weak incident shock wave, caused by a roof or wall collapsing or a large piece of machinery or equipment falling down, propagates through the chamber. The shock wave creates a sudden pressure and density gradient in the background gas. The pressure and temperatures behind this wave are not high enough to cause immediate ignition, however, when this wave reflects from the obstacle (represented by the storage container), the temperatures and pressure increase again, and this results in spontaneous ignition. The numerical simulations are performed from the relatively fundamental approach of solving the compressible equations of fluid dynamics (Euler equations), but with a phenomenological model for species conversion and energy release. The effects of energy release and of the height of the obstacle on the resulting fire severity are explored.

\section{THE MODEL AND METHOD OF SOLUTION}

\section{Fluid Dynamics Model}

The numerical simulation is based on the solutions of the compressible, timedependent, conservation equations for total mass density $\rho$, momentum density $\rho \bar{v}$, and energy density, $\mathrm{E}$,

$$
\begin{aligned}
& \frac{\partial \rho}{\partial t}=-\nabla \cdot \rho \bar{v} \\
& \frac{\partial \rho \bar{v}}{\partial t}=-\nabla \cdot(\rho \bar{v})-\nabla P \\
& \frac{\partial E}{\partial t}=-\nabla \cdot(E \bar{v})-\nabla \cdot(\bar{v} P)
\end{aligned}
$$

where $\vec{v}$ is the fluid velocity and $\mathrm{P}$ the pressure. In a multispecies fluid in which chemical reactions result in transformations among the species, we also need individual species number densities $\left\{n_{\mathrm{i}}\right\}$,

$$
\frac{\partial n_{i}}{\partial t}=-\nabla \cdot n_{i} \bar{v}+Q_{i}-L_{i} n_{i}, \quad i=1, \ldots, N_{s}
$$

where the $\left\{Q_{i}\right\}$ and $\left\{L_{i}\right\}$ are chemical production and loss terms, respectively, for species $i$. There is a constraint that defines the total number density, $N$,

$N=\sum_{i=1}^{N_{i}} n_{i}$

where $N_{\mathrm{s}}$ is the number of different kinds of species present. The total energy is a sum of the kinetic and specific internal energy, $\epsilon$

$E=\frac{1}{2} \rho \bar{v} \cdot \bar{v}+\varepsilon$

The ideal gas equations of state used for the gas-phase calculations are 
$\varepsilon=P /(\gamma-1)$

where $\mathrm{k}$ is the Boltzmann constant, $\mathrm{R}$ is the universal gas constant, $\mathrm{T}$ is the temperature and $\gamma$ is the ratio of specific heats.

\section{Chemistry Model}

The full details of the chemical reactions are not included in this model. Instead, we use the induction parameter model that reproduces the essential features of the chemical reaction and energy release process. This model is valid for fast flows in which the convective timescales are significantly faster than those for physical diffusion. The model was described originally by Oran et al. [6] and has been developed further by Kailasanath et al. [7] and Guirguis et al. [8,9].

In the induction parameter model, the combustion of a premixed propane-air mixture proceeds by a simplified two-step parametric model. During the first step, the reactants break up and intermediate radicals are formed, but because there is not yet any substantial energy release, the mixture remains essentially thermoneutral. The time corresponding to the first step, the chemical induction period, $\mathfrak{r}_{l}$, is fit to an expression based on the experimental data of Burcat et al. [10],

$\tau_{i}=4.4 \times 10^{-14} \exp \left[\frac{42.2 \times 10^{3}}{R T}\right]\left[C_{3} H_{8}\right]^{0.57}\left[O_{2}\right]^{-1.22}$

where the concentrations are in moles/cc and $\tau$ is in seconds. After $\tau$ is calculated for a particular temperature and concentration, the fraction, $f$, of the chemical induction time that has elapsed at time $t$ is calculated from

$\frac{d f}{d t}=\frac{1}{\tau_{i}}$

The second step models the energy-release process, which is the time of rapid reactions and formation of stable products that starts after the induction time has elapsed. Reactants are converted to products according to the finite reaction rate, $\frac{d \omega}{d t}=-\omega A_{r} \exp \left[\frac{-E_{r}}{R T}\right]$

where $\omega$ is the concentration of fuel, $A_{r}$ is the pre-exponential factor, and $E_{r}$ is the activation energy. During this energy release step, $\mathrm{E}_{\mathrm{r}}$ is considered to be zero, and hence the analytical solution to Eq. 11 is a simple exponential.

The function of the induction parameter is to keep track of the temperature history and determine the chemical changes in each fluid element. When the element is heated long enough, energy release is initiated. During the induction step, the induction parameter represents the fraction of elapsed chemical induction time. During the energy release step, the induction parameter represents the fraction of unreacted fuel. As discussed below, this induction parameter is a Lagrangian 
quantity convected at the fluid velocity, so that it stays with each fluid element. To complete the parametric model, we define the value of the final concentration of the fuel, which is obtained from equilibrium calculations. Specification of the final concentration of the fuel determines the amount of energy released. We have chosen the final concentration such that the burnt mixture temperature is $2000 \mathrm{~K}$, which is typical of a stoichiometric propane-air mixture.

\section{Numerical Method of Solution}

The convective transport equations, those parts of Eqs. (1) - (4) excluding the chemical reaction terms, are rewritten in terms of finite-difference approximations on an Eulerian grid. The mass density, momentum density, and total energy density are convected using the nonlinear, fully compressible, flux corrected transport (FCT) algorithm [11], LCPFCT [12, 13]. The induction parameter is also convected with the fluid, so that it stays with the fluid element. FCT is an explicit, finite-difference algorithm with fourth-order phase accuracy and is designed to ensure that all conserved quantites remain monotonic and positive. The procedure for using this one-dimensional algorithm with direction and timestep splitting to produce twodimensional or three-dimensional solutions is described in some detail by Oran and Boris [12]. Those parts of the coupled set of equations that describe the chemical reactions, Eqs. 10 and 11, are solved analytically. These results are combined with the FCT solutions for the convective transport by timestep-splitting methods, as discussed in [12].

Simulations were conducted on a two-dimensional Cartesian grid with 200 cells in the $x$-direction and 30 cells in the $y$-direction. The computational domain is shown in Figure 1. The grid spacing was uniform with $8 x=8 y=0.5 \times 10^{-2} \mathrm{~m}$. The obstacle was located at cell 50 along the $\mathrm{x}$-axis, and is five cells $\left(2.5 \times 10^{-2} \mathrm{~m}\right)$ wide and 15 cells $\left(7.5 \times 10^{-2} \mathrm{~m}\right)$ high. Timesteps used in the simulations are on the order of 1 $x 10^{-6} \mathrm{~s}$. The inflow boundary condition [13] specifies the density, temperature, pressure, and velocity of the inflowing gas, which are determined from the normal shock relations for an ideal planar shock with a Mach number of 1.34. The righthand side of the computational domain boundary has an extrapolated outflow boundary condition [13], which ensures that the fluid properties do not change

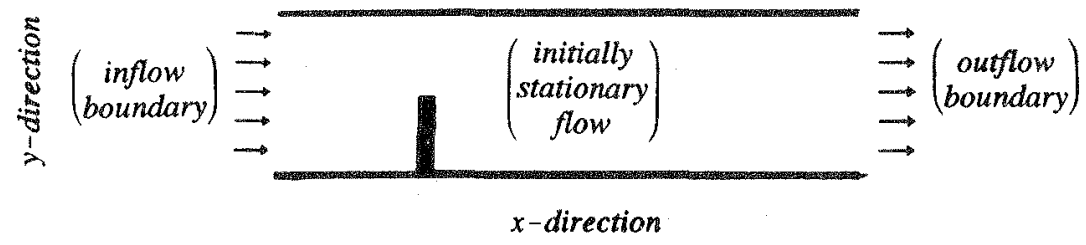

Figure 1. Computational domain. Inflow boundary condition on left-hand side, outflow boundary condition on right-hand side. Top and bottom surfaces are solid walls. The solid vertical rectangle represents the storage container. 
between the last cell inside the computational domain and the guard-cell outside the domain. The top and bottom boundaries are solid walls, represented by free-slip boundary conditions [13].

In these simulations, the initial conditions represent a situation in which the propane gas has already leaked from the storage container and is uniformly distributed in the chamber. The simulation begins with the introduction of the vertical planar shock wave at the inflow boundary. Diffusion of propane into the surrounding gas is therefore completed before the simulation begins.

\section{RESULTS}

Listed in Table 1 are the simulations conducted for this study. Case A-1 is a nonreactive simulation for air and shows the flow resulting from the reflection of a shock wave off of an obstacle. Cases B1 through B-3 are reactive flow simulations of a stoichiometric propane-air mixture. In all cases, the ambient background gas is at standard conditions (STP), and the incoming shock is travelling at Mach (M) 1.34.

TABLE 1. Listing of simulations conducted for this study

\begin{tabular}{llll}
\hline Case & $\begin{array}{l}\text { Incoming } \\
\text { Shock }\end{array}$ & $\begin{array}{l}\text { Background } \\
\text { Gas }\end{array}$ & $\begin{array}{l}\text { Obstacle } \\
\text { Height }(\mathrm{m})\end{array}$ \\
\hline A-1 & Air, M=1.34 & Propane-Air, STP & $7.5 \times 10^{-2}$ \\
B-1 & Propane-Air, M=1.34 & Propane-Air, STP & $7.5 \times 10^{-2}$ \\
B-2 & Propane-Air, M=1.34 & Propane-Air, STP & $3.5 \times 10^{-2}$ \\
B-3 & Propane-Air, M=1.34 & Propane-Air, STP & $11.5 \times 10^{-2}$ \\
\hline
\end{tabular}

\section{Case A-1: Nonreactive Case}

Figure 2, the pressure contours for time steps 80 through 260 , shows the basic structure that evolves as the planar, vertical shock passes over the obstacle. If there were no obstacle, the shock would continue unperturbed through the chamber, slowed only by viscous losses in the system. However, the presence of the obstacle partially blocks the flow and drastically changes the flow both qualitatively and quantitatively. At step 80 , the shock front has just reached the obstacle surface. By step 100, the lower half of the shock front has reflected from the obstacle producing a Mach 2.0 reflected shock. The reflected shock wave moves both upstream (toward the left) and expands upward into the region above the obstacle by step 140. The reflected shock which has expanded above the obstacle reaches the top boundary (solid wall) by step 200, and forms a Mach stem at this upper wall by step 220 . The upper half of the incident shock is transmitted over the obstacle and has cleared it by step 120 . The transmitted shock expands while travelling downstream (toward the right) and reaches the lower solid-wall boundary by step 220 . At step 240, the transmitted shock forms a Mach stem at the lower boundary wall. 


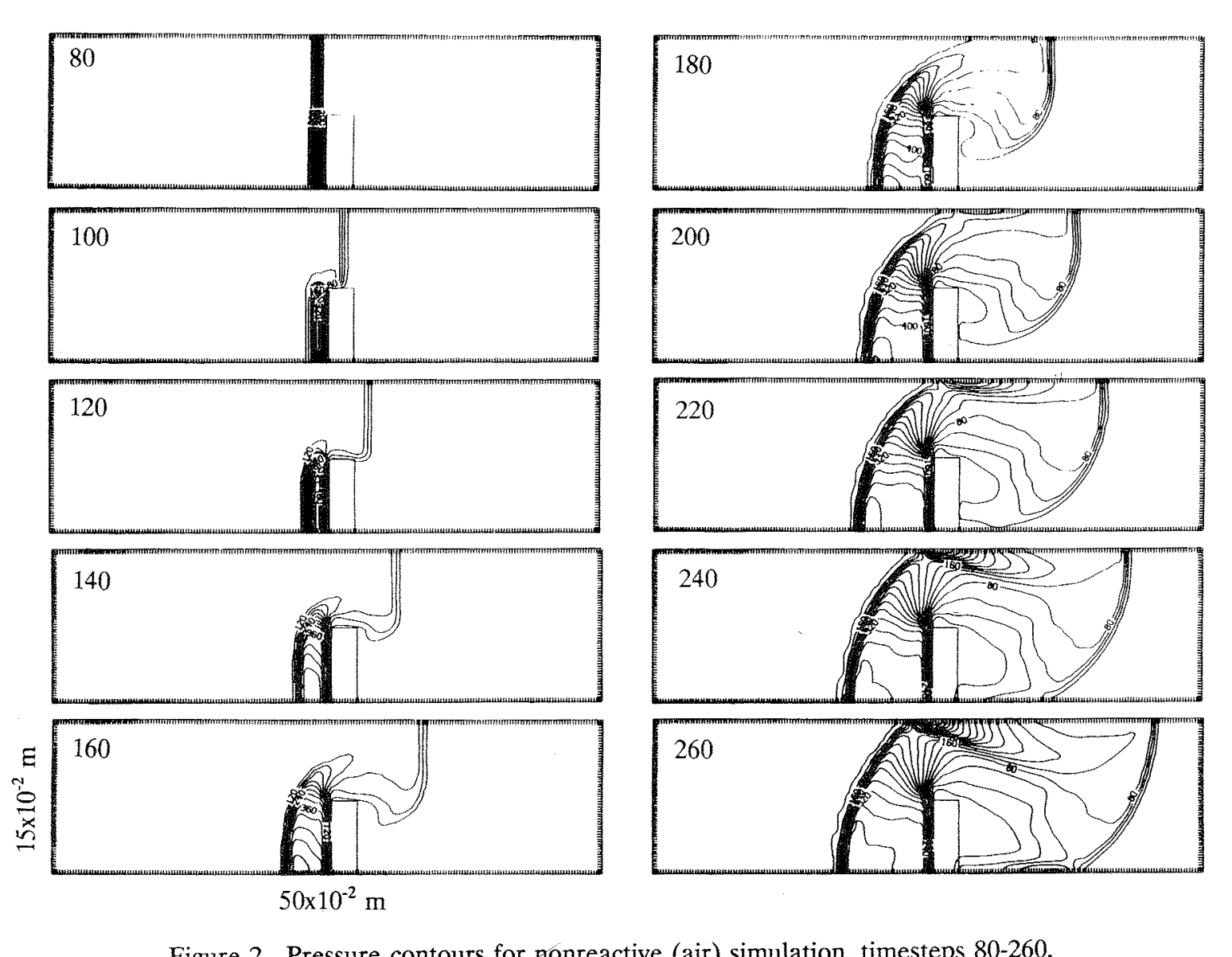


Figures 3 shows the pressure and product contours, respectively for case B-1, which is the same as A-1, but where the mixture is now propane and air that are allowed to react chemically under appropriate conditions. Due to space limitations of this paper, these contours are shown only for timesteps 200 through 280 . As in Case A-1, the lower half of the incident shock front reflects from the obstacle surface by step 100 . The maximum temperature behind the reflected shock is $1350^{\circ} \mathrm{K}$, which corresponds to a chemical induction time of $30 \times 10^{-6} \mathrm{~s}$. Thus by step 140 , energy release has begun in the reflected shock region and products have begun to form. The reaction wave is initiated behind the reflected shock, starting very close to the obstacle, and is closely coupled with the reflected shock front. By timestep 180, the reaction wave behind the obstacle has caught up with the reflected shock front, causing it to transition to a detonation.

This detonation moves upstream and expands upwards into the region above the obstacle. As shown in Figure 3, the detonation reaches the top wall boundary by timestep 200 . When the detonation reflects off of the top wall boundary, it generates a detonation travelling upstream, another detonation travelling downstream, and also generates a shock wave that moves downward towards the obstacle. At timestep 240, Figure 3 shows a detonation travelling upstream. The detonation travelling downstream appears as a curved detonation, and the shock wave that moves down towards the obstacle is attached to the curved detonation moving downstream into the unreacted propane-air mixture.

The downward-travelling shock wave reaches the lower boundary wall by timestep 280. When this shock reflects from the lower wall, it creates a reaction front at this location. This reaction front is attached to the curved detonation (that is travelling downstream) by an oblique shock wave, as shown at timestep 280. This reaction front moves upstream to consume any unreacted propane, and also moves downstream with the attached detonation. This united detonation front eventually overtakes the original transmitted shock which passed over the obstacle.

\section{Cases B-1 through B-3: Effect of Obstacle Size}

To determine the effect of obstacle size on the resulting detonation, Case B-1 simulations were repeated with obstacle heights of $3.5 \times 10^{-2} \mathrm{~m}$ and $11.5 \times 10^{-2} \mathrm{~m}$, respectively. Figure 4 shows the pressure and product contours at a much later time, step 400 . The contours for the $3.5 \times 10^{-2} \mathrm{~m}$ and $7.5 \times 10^{-2} \mathrm{~m}$ obstacles show that the reaction front has caught up with the transmitted shock front and hence has transitioned to a detonation by this time. However, the transmitted shock which passed over the $11.5 \times 10^{-2} \mathrm{~m}$ obstacle had to travel comparatively further before it reflected from the bottom wall, and hence was much weaker when it hit this bottom wall. For the $11.5 \times 10^{-2} \mathrm{~m}$ obstacle, there is a detonation in the lower half of the figure, however, the upper portion has not yet transitioned. The full transition to detonation does occur, however, by 500 timesteps for the tallest obstacle. 


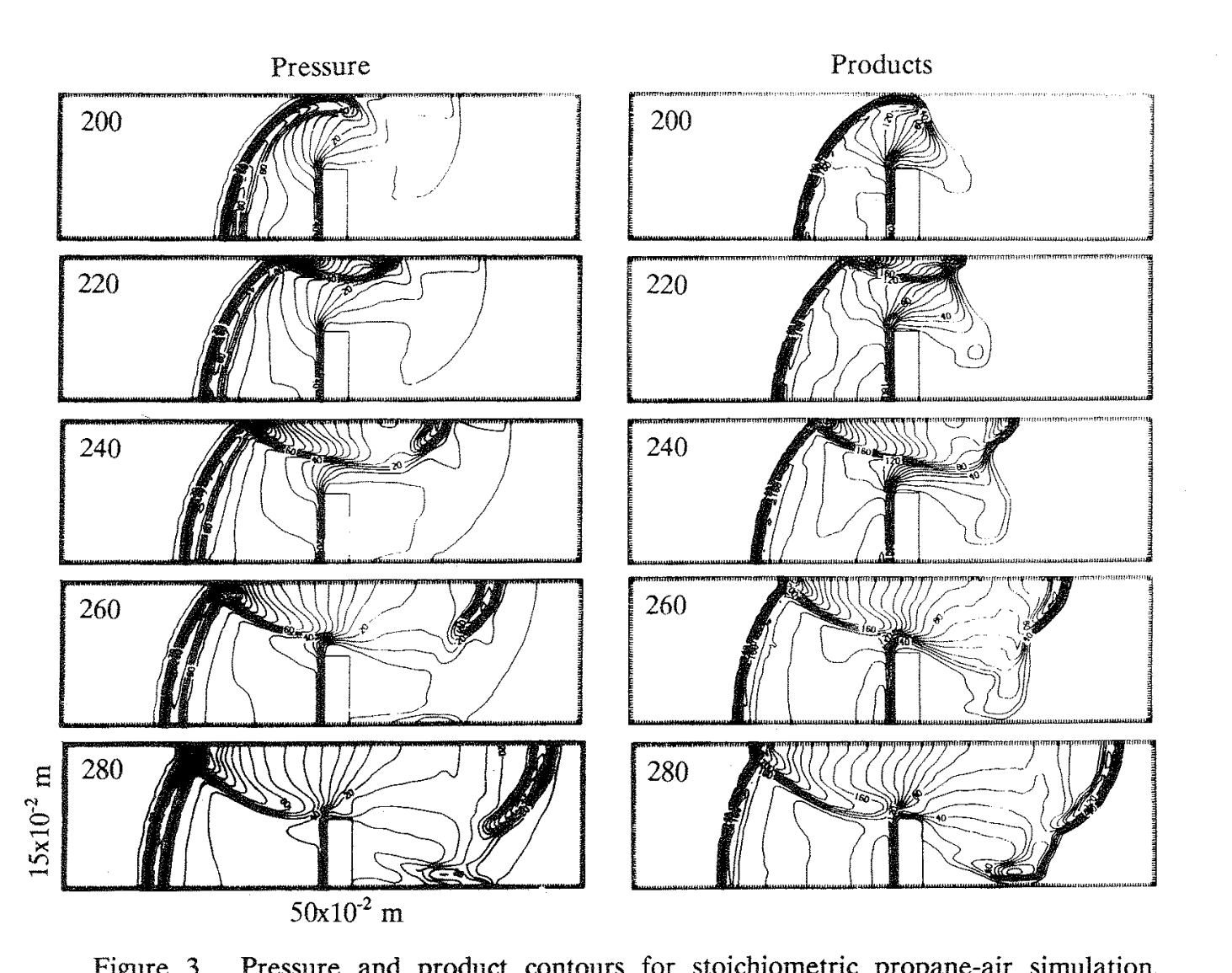



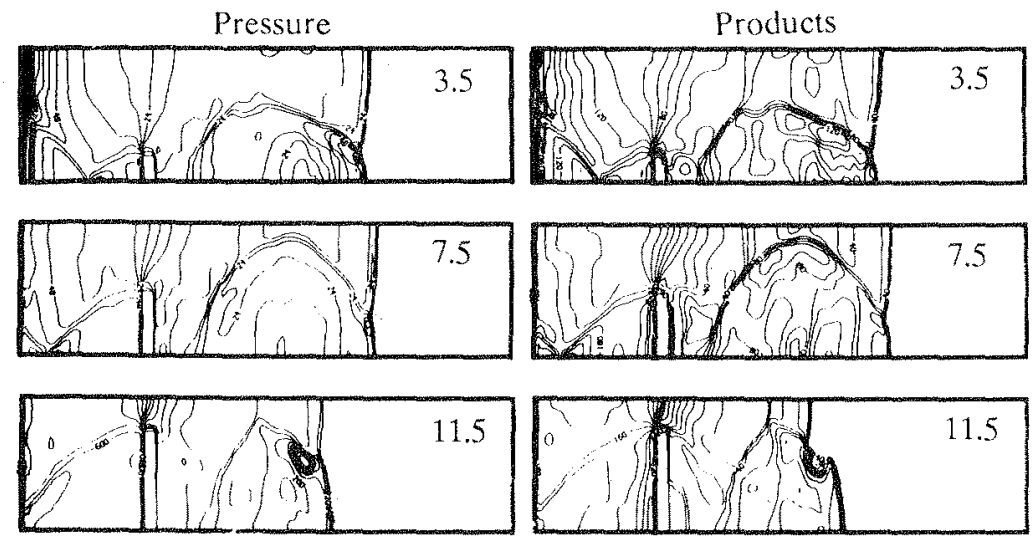

Figure 4. Pressure and product contours at timestep 400, for obstacles of height $3.5 \times 10^{-2} \mathrm{~m}, 7.5 \times 10^{-2} \mathrm{~m}$, and $11.5 \times 10^{-2} \mathrm{~m}$.

\section{OBSERVATIONS}

In the previous section, the spontaneous ignition of a stoichiometric propaneair mixture began after a weak shock reflected from an obstacle. The shock reflection resulted in the formation of a reaction wave behind the reflected shock, which quickly transitioned to a detonation moving upstream, and eventually transitioned to a detonation travelling downstream. Computations performed to evaluate the effect of changing the relative height of the obstacle showed that, in every case studied, the transmitted shock transitioned to detonation; it simply took longer for this transitioin to occur when the obstacle was the tallest.

The initial conditions of the simulations presented above were such that the propane gas had leaked from a storage container and was uniformly distributed in the simulated area before the introduction of the planar shock wave. Further computations [14] present a more realistic physical scenario, in which the fuel concentration was greatest near the storage container $(\phi=2)$ and proportionately decreased with distance from the container to a fuel lean mixture $(\phi=0.5)$ at the boundary. These variable stoichiometry simulations [14] showed that a reaction wave was initiated in the reflected shock region, but was confined to this region. As the transmitted shock propagated downstream and into the stoichiometric region, a new ignition source developed, which transitions to detonation when reflecting from the lower wall boundary. However, as this detonation propagated through a progressivly leaner mixture further downstream, it decayed into a shock wave followed by a decaying flame front.

\section{ACKNOWLEDGEMENTS}

This work was sponsored by the Naval Research Laboratory through the Office of Naval Research. The authors would like to thank Dr. K. Kailasanath for 
assistance with the chemical induction model, and Drs. F. Willimas, P. Tatem and J. Boris for providing the resources and environment necessary for this work to be accomplished.

\section{REFERENCES}

1. Moen, I.O., Sulmistras,A., Hjertager, B.H., and Bakke, J.R., "Turbulent Flame Propagation and Transition to Detonation In Large Fuel-Air Clouds," Proceedings of the Twenty-First Symposium (International) on Combustion, The Combustion Institute, Pittsburgh, PA, p. 1617 (1986).

2. Urtiew, P.A., Brandeis, J. and Hogan, W.J., "Experimental Study of Flame Propagation in Semiconfined Geometries with Obstacles," Combust. Sci. Tech., 30 , 105 (1983).

3. Moen, I.O., Lee, J.H.S., Hjertager, B.H., Fuhre, K., and Eckhoff, R.K., "Pressure Development Due to Turbulent Flame Propagation in Large-Scale Methane-Air Explosions," Combust. Flame, 47, 31 (1982).

4. Taylor, P.H., "Fast Flames In a Vented Duct," Proceedings of the Twenty-First Symposium (International) on Combustion, The Combustion Institute, Pittsburgh, PA., p. 1601 (1986).

5. Chan, C., Moen, I.O. and Lee, J.H.S., "Influence of Confinement on Flame Acceleration Due to Repeated Obstacles," Combust. Flame, 49, 27 (1983).

6. Oran, E.S., Boris, J.P., Young, T.R. and Picone, J.M., "Numerical Simulations of Detonations in Hydrogen-Air and Methane-Air Mixtures," Proceedings of the Eighteenth Symposium (International) on Combustion, The Combustion Institute, Pittsburgh, PA, p. 1641 (1981).

7. Kailasanath, K., Oran, E.S., and Boris, J.P., "Determination of Detonation Cell Size and the Role of Transverse Waves in Two-Dimensional Detonations," Combust. Flame, 61, 199 (1985).

8. Guirguis, R., Oran, E.S., and Kailasanth, K., "Numerical Simulatons of the Cellular Structure of Detonations in Liquid Nitromethane - Regularity of the Cell Structure," Combust. Flame, 65, 339 (1986).

9. Guirguis, R., Oran, E.S., and Kailasanath, K., "The Effect of Energy Release on the Regularity of Detonation Cells in Liquid Nitromethane," Proceedings of the Twenty-First Symposium (International) on Combustion, The Combustion Institute, Pittsburgh, PA 11987, p. 1659 (1987).

10. Burcat, A., Lifshitz, A., Scheller, K., Skinner, G.B., "Shock Tube Investigation of Ignition in Propane-Oxygen Argon Mixtures, Proceedings of the Thirteenth Symposium (International) on Combustion, The Combustion Institute, Pittsburgh, PA., p. 745 (1971).

11. Boris, J.P. and Book, D.L., Methods in Computational Physics, 16, 85 (1976).

12. Oran, E.S. and Boris, J.P., Numerical Simulation of Reactive Flow, Elsevier, New York, NY (1987).

13. Boris, J.P., Gardner, J.H., Oran, E.S., Zalesak, S., Ellzey, J., Patnaik, G. Book, D.L., Guirguis, R.H., LCPFCT - A Monotone Algorithm for Solving Continuity Equations, to appear, Naval Research Laboratory Memorandum Report (1990).

14. Kaplan, C.R. and Oran, E.S., "Spontaneous Ignition and Detonation of PropaneAir Mixtures," submitted to Combust. Sci. Tech. 